\title{
Convolution Neural Network Application in Kidney Tumor Segmentation on CT Images
}

\author{
Jianping Hunag ${ }^{1}$, Zefang Lin $^{1}$ \\ ${ }^{1}$ South China Normal University \\ \{jianping, 2017021649$\} \mathrm{em}$. scnu. edu.cn
}

\begin{abstract}
In this paper, we propose an novel network model which is similar to $\mathrm{V}$-net and prove its superiority and efficiency in tumor segmentation. And The model of segmentation of Kidney is Dense V-Network [1]. Then we ensemble the results of two networks together to get a final predict result for kidney and tumor. In particularly, we apply a series of method to image preprocessing, which is proved to be effective in improving dice.
\end{abstract}

Keywords: Convolution Neural Network, Preprocess method, Tumor Segmentation.

\section{$1 \quad$ Preprocess}

\section{$1.1 \quad$ Pre-cut}

In order to reduce useless context information away from the kidney area, we cropped the original images into images with the shape of $372 \times 372$ on transverse plane and the cropped result is followed as Fig. 1. It can be seen from Fig.1 that the major context information is still exist.

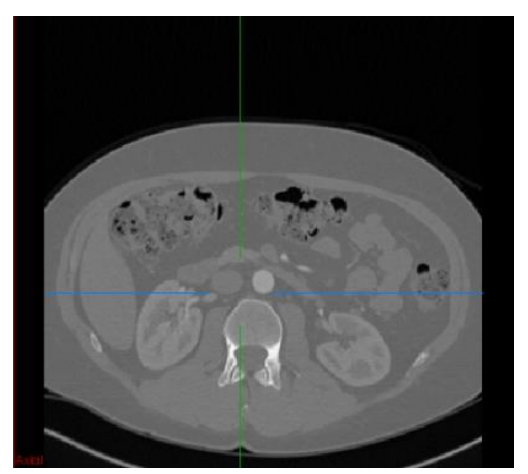

Fig. 1. The cropped image 


\subsection{Resize}

We resize the cropped CT image into the images with the shape of $[240,240,184]$ to make it to be suitable for the input shape of Dense V-Network. $(240 \times 240$ is the shape of transverse plane and the 184 is the number of layers).

\subsection{Combine Labels}

The first convolution neural network is used to obtain the combined area of kidney and tumor. Thus we combine two classes label to be one class label.

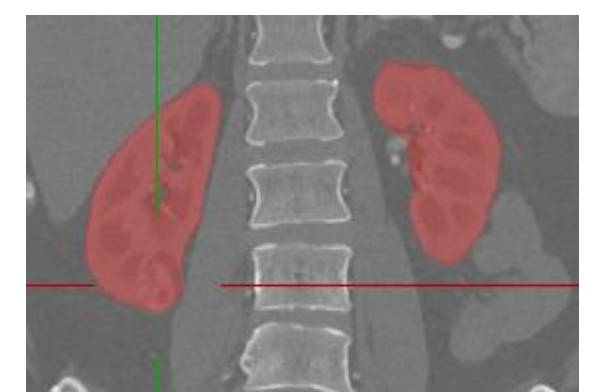

Fig. 2. The one class label after combing kidney and tumor classes label.

\section{Segmentation of Kidney}

We select Dense V-Network to segment the kidney for its good performance in automatic multi-organ segmentation. The implementation is performed on NiftyNet [2] and the result of segmentation can be seen from Fig. 3 .

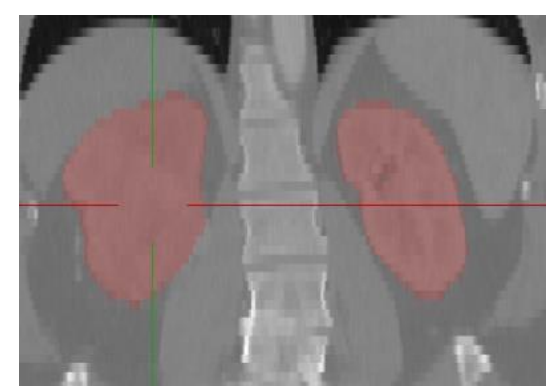

Fig. 3. The segmentation result of Dense V-Network on test set. 


\section{Segmentation of Tumor}

\subsection{Obtaining the ROI of Tumor}

In order to reduce useless context information for segmentation of tumor, we crop the cropped CT images again according to the output of Dense V-Network and thus the ROI of tumor can be obtained.

We set a fixed value for each pixel that not in mask acquired by the output of Dense V-Network because the kidney tumor grows in the kidney and from the output of Dense V-Network, the area of kidney included the area of tumor.

The model used to segment the tumor include 5 down-samples. We resize the ROI of tumor to $[128,256,160](128 \times 256$ is the shape of transverse plane and the 160 is the number of layers).

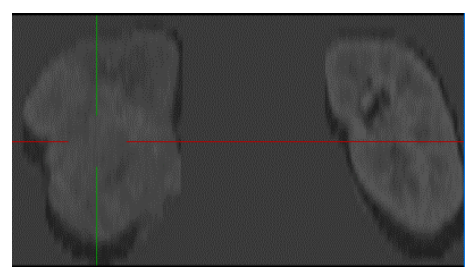

Fig. 4. The ROI of tumor on test set.

\subsection{Attention V-Net}

We referenced the structure of Attention U-Net [3] and V-Net [4] implement Attention V-Net. In the part of up sample of V-Net, we introduced the attention-gate into the model and the structure of attention gate can be seen from Fig. 5.

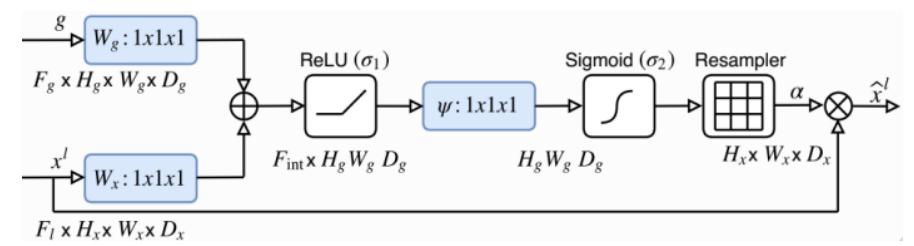

Fig. 5. The structure of attention gate.

\section{$4 \quad$ Post Process}

We use open operations on the output to remove noise. Then we combine the results from two models to get the final result. The final result is followed as Fig.6. 


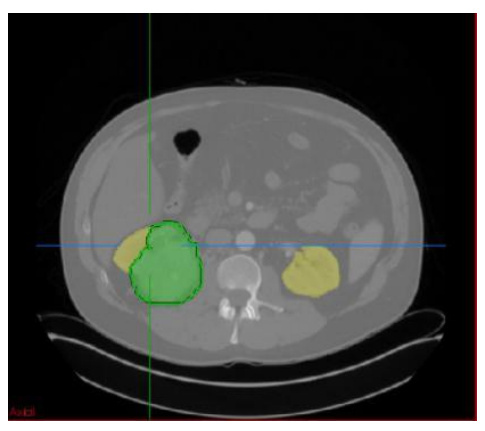

Fig. 6. The final output.

\section{References}

1. Gibson et al.: "Automatic Multi-Organ Segmentation on Abdominal CT with Dense V-Networks," in IEEE Transactions on Medical Imaging, vol. 37, no. 8, pp. 1822-1834, Aug. 2018.

2. Gibson et al.: "NiftyNet: a deep-learning platform for medical imaging". In: Computer Methods and Programs in Biomedicine Volume 158, pp. 113-122, May 2018.

3. Oktay et al.: " Attention U-Net: Learning Where to Look for the Pancreas ". In: ArXiv 2018.

4. Milletari et al.: " V-Net: Fully Convolutional Neural Networks for Volumetric Medical Image Segmentation". In: 2016 Fourth International Conference on 3D Vision (3DV), Stanford, CA, 2016, pp. 565-571. 